\title{
The Emergence of Symbol-Based Communication in a Complex System of Artificial Creatures
}

\author{
Angelo Loula ${ }^{1}$, Ricardo Gudwin ${ }^{1}$, Charbel Niño El-Hani ${ }^{2}$, and João Queiroz ${ }^{1,2, *}$ \\ ${ }^{1}$ DCA, FEEC, State University of Campinas, Brazil. \\ ${ }^{2}$ Institute of Biology, Federal University of Bahia, Brazil. \\ * Corresponding Author: queirozj@dca.fee.unicamp.br
}

\begin{abstract}
We present here a digital scenario to simulate the emergence of self-organized symbol-based communication among artificial creatures inhabiting a virtual world of predatory events. In order to design the environment and creatures, we seek theoretical and empirical constraints from C.S.Peirce Semiotics and an ethological case study of communication among animals. Our results show that the creatures, assuming the role of sign users and learners, behave collectively as a complex system, where selforganization of communicative interactions plays a major role in the emergence of symbol-based communication. We also strive for a careful use of the theoretical concepts involved, including the concepts of symbol, communication, and emergence, and we use a multi-level model as a basis for the interpretation of inter-level relationships in the semiotic processes we are studying.
\end{abstract}

\section{INTRODUCTION}

In this paper, we report the simulation of an ecosystem that allows cooperative interaction between distributed agents, including intra-specific communication, a process that can raise the fitness of individuals in the face of predatory events. As part of a project dealing with the emergence and grounding of symbols, we adopt Peirce's theory of signs as a theoretical framework to define semiotic processes and categories (e.g., meaning, symbol) as well as to develop simulations inspired by a case study of communication among vervet monkeys. We also strive for a careful use of the theoretical concepts involved, including the concept of 'emergence'. Furthermore, we interpret our results on the grounds of a multi-level model for explaining the emergence of semiosis in semiotic systems, which was previously developed in $[1,2]$.

\section{C.S. PeirCe's Semiotics}

Semiosis (meaning process) can be characterized as a pattern of behaviors that emerges through the intra/intercooperation between agents in a communication act. According to C.S.Peirce, it involves a self-corrective process whose structure exhibits an irreducible relation between sign, object and interpretant.

“... a Sign may be defined as a Medium for the communication of a Form. [...]. As a medium, the Sign is essentially in a triadic relation, to its Object which determines it, and to its Interpretant which it determines. [...]. That which is communicated from the Object through the Sign to the Interpretant is a Form; that is to say, it is nothing like an existent, but is a power, is the fact that something would happen under certain conditions" (EP 2. 544) [3].

Signs, objects, and interpretants constitute, thus, an irreducible triadic relation (or triad). The action of a sign, i.e., semiosis, entails, however, the instantiation of chains of triads. An interpretant is both the third term of a given triadic relation and the first term (sign) of a subsequent triadic relation. This is the reason why semiosis cannot be defined as an isolated triad; it necessarily involves chains of triads [2].

There are three fundamental kinds of signs underlying meaning processes - icons, indexes, and symbols. This corresponds to the 'most fundamental division of signs' (CP 2.275) [4] based on the nature of the relation between S-O (sign-object). Icons are signs that stand for their objects through similarity or resemblance (CP 2.276) [4]. In an index, $\mathrm{S}$ and $\mathrm{O}$ are related through 'a direct physical connection' (CP 1.372) [4] and "the interpreting mind has nothing to do with this connection, except remarking it, after it is established" (CP 2.299) [4]. Finally, if the relation between $\mathrm{S}$ and $\mathrm{O}$ logically depends on a law-like mediation by a third element, the interpretant (I), $\mathrm{S}$ is a symbol of $\mathrm{O}$.

\section{THE MEANING OF EMERGENCE}

We claim that the digital scenario we propose here allows us to simulate the emergence of self-organized symbol-based communication among artificial creatures. We should use the term 'emergence' and its derivatives (such as 'emergent property') in a technical sense in the sciences of complexity, although this is not often the case [1]. In this sense, emergent properties or processes can be defined as a certain class of higher-level properties or processes related in a certain way to the microstructure of a class of systems. Then, an emergence theory should be proposed to fill in the open clauses in this definition (shown in italics), providing, among other things, an account of which systemic properties or processes of a class of systems are to be regarded as 'emergent' and offering an explanation of how they relate to the microstructure of such systems. We can develop such a theory by answering questions which follow from a 
systematic analysis of emergence theories and concepts [1], such as that offered by Stephan [5,6]. Even though we refer our readers for our previous paper quoted above for a more detailed presentation of these questions and their respective answers, we will briefly present here the main features of the emergence theory we proposed on their grounds.

Symbol-based communication is a kind of semiotic process, and, thus, the first constraint for a system capable of exhibiting such an emergent process is that it should be a semiotic system. A semiotic system is a system that produces, transmits, receives, computes, and interprets signs of different kinds [7]. This kind of system is capable of symbol-based communication when the signs it handles are in a law-like triadic relation to the object mediated by a third term, the interpretant, which stands for the object through the sign (see [1]).

In a previous paper [1, 8], we took Salthe's [9] basic triadic system (Figure 2) as a ground for developing a three-levels model for semiotic systems/processes. In this model, we consider (i) a focal level, where an entity or process we are interested in is observed in a hierarchy of levels; (ii) a lower level, where we find the parts composing that entity or process; and (iii) a higher level, in which the entities or processes observed at the focal level are embedded. What emerges in a semiotic system is the product of processes at the focal level that are instantiated through the interaction between processes taking place at the next lower and higher levels, i.e., between the relations of determination within each triad and the embedment of each individual chain of triads in a whole network of sign processes. We can talk about 'micro-semiosis' when we refer to a repertoire of potential signs, objects, and interpretants, which might be involved in relations of determination so as to form triads. When triads are effectively formed, we can talk about 'focal-level semiosis', which necessarily involve a chain of triads, as explained above. But the actualization of a triad from the repertoire of potential signs, objects, and interpretants depends on a macro-semiotic level, containing networks of chains of triads, in which each individual chain is embedded.

Symbol-based communication should be regarded as a systemic process (and, therefore, is indeed a candidate for being an emergent process) because, as we just saw, the actualization of potential triads depends on boundary conditions established by a macro-semiotic level, amounting to networks of chains of triads. Therefore, although symbolbased communication is instantiated, according to our model, at the focal level, it is indeed a systemic process, as the macro-semiotic level establishes the boundary conditions required for its actualization.

Another important requirement for semiosis is that semiotic processes can only be realized through physical implementation or instantiation, and, thus, any semiotic system, including those capable of handling symbols, should be physically embodied.
Emergentist thinking is also characterized by a fundamental commitment to the notion of novelty. Accordingly, we adopt here an epigenesis view about the origin of systems capable of producing, transmitting, receiving, computing, and interpreting signs. We can say in these terms that semiotic systems constitute a new class of systems, with a new kind of structure, capable of producing and interpreting signs, and, thus, of realizing semiosis, as an emergent process.

Another characteristic notion of emergence theories that we should take into account is the thesis of synchronic determination, according to which a system's properties and behavioral dispositions depend on, and is determined by, its microstructure, i.e., its parts' properties and arrangement. To examine the idea of synchronic determination, we have to focus our attention on the relationship between chains of triads and individual triads. It is clear from the Peircean framework that all kinds of semiosis are synchronically determined by the microstructure of individual triads composing a chain of triads, i.e., by the relational properties and arrangement of the elements $\mathrm{S}, \mathrm{O}$, and $\mathrm{I}$.

We should also consider whether and in what sense semiosis can be treated as an irreducible and/or unpredictable process. The semiotic triadic relation is regarded by Peirce as irreducible in the sense that it is not decomposable into any simpler relation (see, e.g., CP 5.484). According to Peirce, if we consider only a dyadic relation, S-I, S-O or I-O, we cannot deduce how they would behave in a triadic relation, S-O-I (EP 2.391) [3].

The structure of triads and chains of triads can be also regarded as in principle theoretically unpredictable in a Peircean framework, since Peirce held the view that an element of indeterminism is present in the natural world. Then, the behavior of the elements in a semiotic process is unpredictable from their behavior in simpler systems, and, consequently, so is symbol-based communication

\section{Simulating Communicative Creatures}

In building the experimental setup, we considered constraints following from biological motivations, inspired by ethological case studies of intra-specific communication for predator warning. More specifically, we examined alarm calls from vervet monkeys. These primates use vocal signs for general alarm purposes regarding imminent predation on the group. This well-studied case of communication for predator warning in vervet monkeys inspired the creatures design and the ecological conditions in our experiment. The creatures are autonomous agents inhabiting a virtual bidimensional environment. ${ }^{1}$

The virtual world is composed of creatures divided into preys and predators (terrestrial, aerial and ground predators), and also of things such as trees (climbable objects) and bushes (used to hide). We have previously proposed two

\footnotetext{
${ }^{1}$ Check www.dca.fee.unicamp.br/projects/artcog/symbcreatures to see the simulator and further technical details.
} 
different roles for preys: teachers (sign vocalizers) and learners (sign apprentices), with teachers emitting predefined alarms for predators and learners trying to find out without explicit feedback with which predators each alarm is associated. In the present paper, we ask what would happen if there were no previous alarm calls and the creatures needed to create their own repertoire of alarms. We introduce a special type of prey, which is a teacher and a learner at the same time, able to create, vocalize, and learn alarms, even simultaneously. These preys are called here self-organizers, because each prey learns the sign it hears and uses them in future interactions, permitting a circular relation to happen: the effect preys have on one another is also the cause of this effect, because sign learning depends on sign usage, which in turn depends on sign learning. The aim of the experiment is to observe the self-organizing dynamics of signs and verify if, starting with no specific signs to predators, a common repertoire of symbol-based alarm calls can emerge via local communicative interaction.

The creatures have sensors and motor abilities that allow their interaction with the virtual environment. The sensorial modalities found in the preys include hearing and seeing, but for the sake of simplicity predators can see but not hear. The creatures also have interactive abilities defined by a set of possible individual actions - adjustment of sensors, movement, attack, climb on tree, hide under bush, and vocalize alarms. The last three actions are specific for preys, while attacks are specific for predators. To perform the connection between sensors and actuators, the creatures make use of a control architecture inspired by behaviorbased approaches (Figure 1).

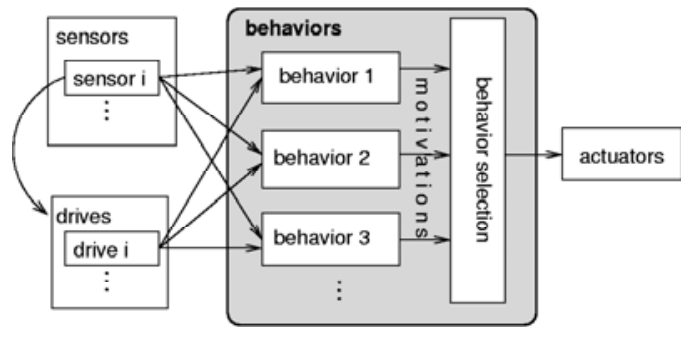

Figure 1: Creatures' general architecture of behaviors, motivations and drives.

The control mechanism used by the creatures is composed of drives, motivations, and behaviors (figure 4). The predator has a simple control architecture with three basic behaviors - wandering, prey chasing, and resting - and two drives - hunger and tiredness. The preys are involved in communicative acts, vocalizing, interpreting and learning alarms. Some of the preys' behaviors, such as vocalizing, scanning, following, along with associative learning, give them the ability to engage in communicative acts. Besides communicating, the preys should also have other tasks to perform (basic behaviors) in order to keep them busy even when not communicating: wandering, fleeing, and resting.
Related to these behaviors, the preys have different drives: boredom, tiredness, fear, solitude, and curiosity. ${ }^{2}$

The behavior of 'following' makes the preys stay together, trying to follow each other. When a prey hears an alarm, the 'scanning' behavior is activated and makes the prey direct its vision to the alarm emitter and its surroundings. The vocalizing behavior makes the prey produces an alarm, when it sees a predator, which can be heard by any other prey, provided the alarm call is within its hearing range. Self-organizers don't have a pre-defined repertoire of alarmpredator associations, and, thus, their vocalizing repertoire depends on associative memory. When a predator is seen, they use the alarm with the highest association strength to that predator, or create a new alarm if none is known. Alarms are created by randomly choosing one among the possible alarms that preys can emit. Running simultaneously with all other behaviors, associative learning is the most important behavior in the experiment.

Associative learning allows the prey to learn temporal and spatial relations from the external stimuli and thus acquire association rules necessary to interpret signs as symbols. Sensorial data from vision and hearing are received by the respective work memories, which are temporary repositories of sensorial stimuli, making it possible that stimuli received in different instants coexist for some time in the memory, preserving indexical relations. Following Hebbian learning principles, when sensorial data enters the work memories, the associative memory creates, or reinforces, the association between the visual item and the hearing item. When an item is dropped from the work memory, related associations can be weakened, if it was not already reinforced. These positive (reinforcement) and negative (weakening) adjustment cycles in the associative memory allow preys to self-organize their repertoire, and common alarm-predator associations to emerge.

The associative learning mechanism also provides a feedback when a vocalization associated with a predator is heard. This produces an internal stimulus of the related predator, and depending on the association strength, an escape response can be elicited. At first, the prey responds indexically to an alarm call through the visual scanning behavior, which helps the learning process. But after the association between alarm and predator gets near maximum value, the internal feedback can activate the fleeing behavior, even if a predator is not seen. Thus at this optimum value the prey stops scanning after an alarm is heard, and flees right away. This characterizes a new action rule, corresponding to an evidence of the alarm becoming a symbol.

\footnotetext{
${ }^{2}$ For further details about the control architecture of preys and predators, see [11].
} 


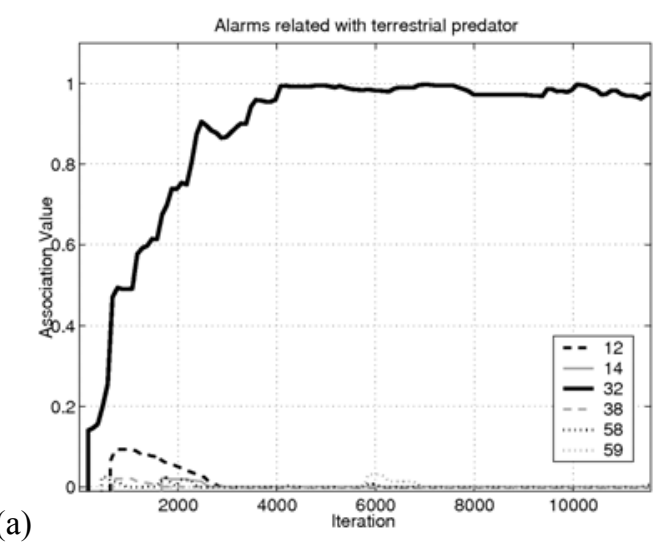

(a)

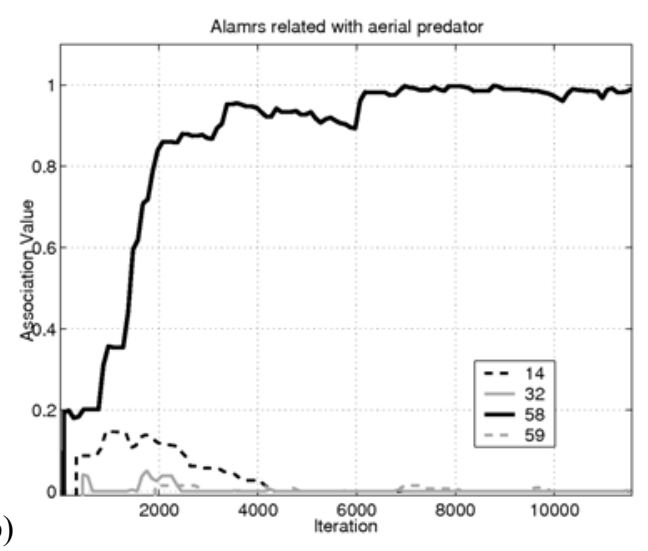

(b)

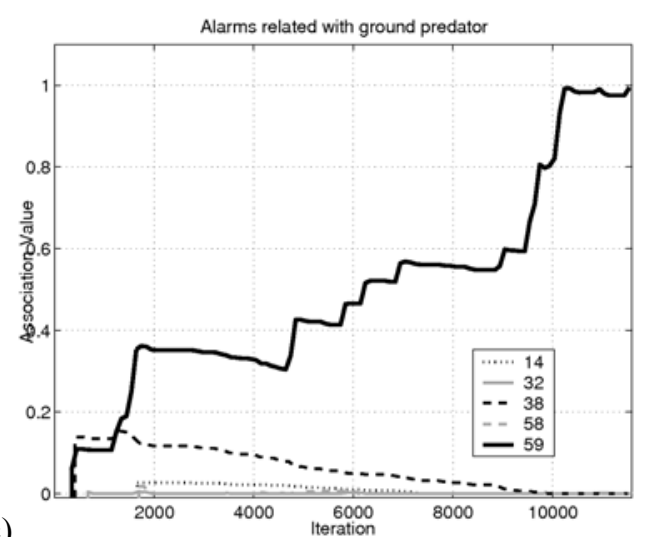

(c)

Figure 2: The mean association values of the alarm-referent associations for 4 self-organizers: (a) terrestrial predator, (b) aerial predator, (c) ground predator.

\section{Creatures in Operation}

In order to study the self-organizing and emergent dynamics in communicative acts, we performed experiments by placing together preys and predators in the environment. During the execution of the simulations, we observed the associative memory items and the behavior responses of the preys to alarm calls. Results show that there was a convergence to a common repertoire of associations between alarms and predators. This is a repertoire of
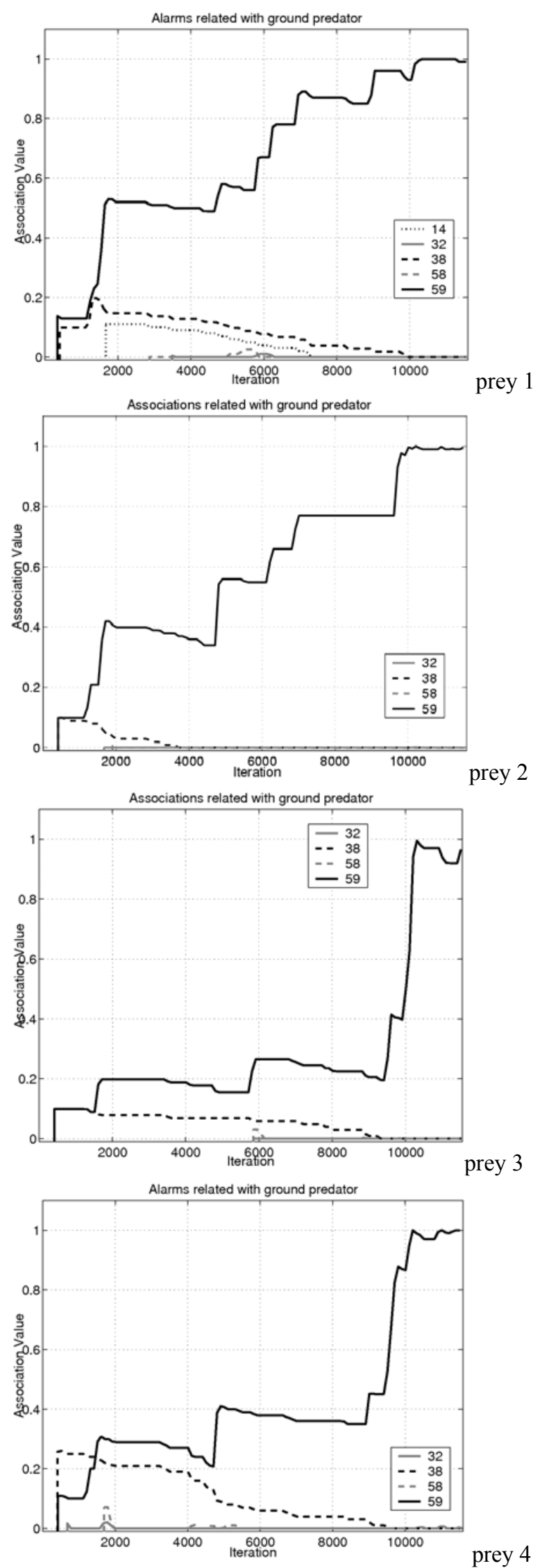

Figure 3: The individual association values of the associations between alarms and ground predator. 
symbols that make the preys engage in escape responses when an alarm is heard, even in the absence of visual cues.

Here we present results from a typical simulation, using 4 self-organizers and 3 predators. The self-organizers can create alarms by randomly selecting one out of 100 possible alarms (from 0 to 99), when no alarm is known for a predator. We let the simulation run until the community of preys converged to a common sign repertoire for the predators. Initially none of the preys have alarms associated with predators. Therefore, at the beginning of the simulation, new alarms are randomly created when they meet predators. This creates an explosion in the available alarms, that tends to be in greater number than the existing predator types. In figure 2, we see that various alarms were created to refer to each predator at first, but soon they stop appearing because every prey will know at least one alarm for each predator. In the graph shown in figure $2 \mathrm{a}$, the terrestrial predator is associated with alarms $12,14,32,38$, 58 and 59, but only alarm 32 reaches the maximum value of 1.0 , and the competing alarms are not able to overcome it at any time. Similar results were found in the case of alarms $14,32,58$ e 59 associated with aerial predator (figure $2 b$ ): only alarm 58 reached a maximum value. But among the alarms for ground predators (figure 2c), there was a more intense competition, which led to the inversion of positions between alarms 38 and 59 . They were created almost at the same time in the community, and initially alarm 38 had a greater mean value than alarm 59 . But between iteration 1000 and 2000, the association value of alarm 59 overcame the value of alarm 38, which slowly decayed, reaching the minimum value after iteration 9000 .

To better understand what happened in the competition between alarms 59 and 38, we present the individual graphs for each prey (figure 3). In these graphs, we see that the associations evolved in distinct ways. Alarm 59 was created by prey 1 and alarm 38 by prey 4 . Preys 2 and 3 learned these alarms, and they had similar association values before iteration 2000. But notice that prey 2 employed alarm 59 to vocalize, because it was learned first, while prey 3 preferred alarm 38 for the same reason. This led to a situation where each two preys preferred a particular alarm (38 or 59). After iteration 2000, the frequency of usage determined the alarm success, and alarm 59 eventually overcame alarm 38 . If an alarm is heard more often or before another, its chance of success is greater, because it will be reinforced more frequently or before the competing alarms. This was the reason why alarm 59 won the competition and was adopted by all preys.

\section{SELF-ORgANizATION AND EMERGENCE OF SYMBOL-BASED COMMUNICATION}

Together, the self-organizers constitute a complex system ${ }^{3}$, with local interactions of communicative acts. By communicating, a vocalizing prey affects the sign repertoire

\footnotetext{
${ }^{3}$ A complex system is a system composed of many elements with nonlinear local interactions, typically conducting to a global pattern.
}

of the hearing preys, which will adjust their own repertoire to adapt to the vocalized alarm and the context in which it is emitted. Thus, the vocalizing competence will also be affected as it relies on the learned sign associations. This implies an internal circularity among the communicative creatures, which leads to the self-organization of their repertoires. This circularity is characterized by positive and negative feedback loops: the more a sign is used the more the creatures reinforce it, and, as a result, the frequency of usage of that sign increases; in turn, the less a sign is used the less it is reinforced, and consequently its usage is decreased.

In this self-organizing system, a systemic process (symbolbased communication), as much as a global pattern (a common repertoire of symbols), emerges from local (communicative) interactions, without any external or central control. This complex system of communicative creatures can be viewed as a semiotic system of symbolbased communication with three different levels, as discussed in section 3 .

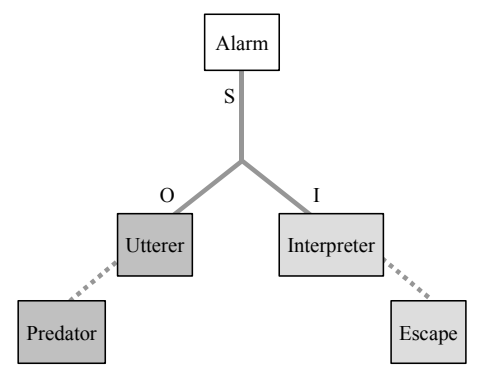

Figure 4: Communication triad relating sign-uttererinterpreter.

The mapping of the proposed triadic hierarchy structure onto our synthetic experiment must be further detailed in order to elucidate the dynamics and emergence of communication events. The focal level corresponds to the communicative phenomena. As described in section 2, Peircean sign model irreducibly relates three elements, signobject-interpretant. This model mainly describes interpretation processes, while here, as we concentrate on communication dynamics, we derive from Peircean triadic model a specific model, which seems to be more adequate, at the present state of our research, to work with communication processes. In this derived model, the object is replaced by an utterer, and the interpretant, by an interpreter (figure 4). More explicitly, we can talk about a vocalizing prey (the utterer) producing an alarm for a hearing prey (the interpreter), trying to transmit a warning escape alert. This communication triad can be connected to a chain of communication events, with the interpreter receiving the meaning embodied in the sign and turning into an utterer of this same form to another interpreter. This implies a possible circularity as mentioned before, when the utterer of the first episode becomes the interpreter at a future event. This succession of triads can become rather complicated if we notice that different utterers can 
communicate with the same interpreter or one utterer can vocalize to different interpreters, both simultaneously.

This focal-level is constrained by a macro-semiotic level of networks of communication triads and a micro-semiotic level of potential sign relations. The micro-semiotic level establishes initiating conditions for communication acts, as it comprises potential signs from 0 to 99 which can be related to any predator by the utterer while, in the case of the interpreter, a potential sign can be associated with any entity in the environment. The environment also plays an essential role in the system dynamics by providing contextual constraints (visual cues). When potential sign relations are actualized, the environment will establish specific constraints for the utterer's sign production (presence of predators) and for the interpreter's sign interpretation (any surrounding entity). The history of focal level processes turns into an interrelated network of communication triads at the macro level, which amounts to the system's history or memory. This history is condensed as the communicative preys develop habits, precisely located in their individual associative memories. Hence, the system's history at the macro level establishes constraints for the system's dynamics which can be treated as boundary conditions, being the system variability reduced with utterers using established signs in its associative memory and interpreters being able to use the same repository to interpret alarms, which ultimately becomes symbols. This macro level is ontologically assembled as communication semiosis starts to take place and exerts a higher-level influence on the focal level as the system evolves. At first, initiating conditions exert a stronger constraining influence on the focal level, as triadic, semiotic relations are created on the grounds of the available potential signs, objects, and interpretants, and a macro-semiotic level is still under construction. As the system's dynamics goes on, the macrosemiotic level constrains more and more the communicative acts actualized at the focal level, and, ultimately, the boundary conditions established by that level guide the system to an ordered state, which amounts to a common repertoire. At this step, symbol-based communication emerges, as a new irreducible property of the semiotic system at stake.

\section{CONCLUSION}

The design and synthesis of the creatures we present here are constrained by meta-principles (such as Peirce's semiotics, emergentist philosophy, the three-levels model for the emergence of semiosis presented in section 3 , and biological motivations. The virtual world we implemented works as a laboratory to simulate the emergence of antipredatory alarm call vocalization among creatures under the risk of predation.

Characterized as a self-organizing system, the community of sign-manipulating individuals is seen as being formed by components interacting in a distributed manner, with emergent global properties, besides an inherent unpredictability and non-linearity. These properties make self-organizing systems hard to be studied by simply analyzing their parts separately. This suggests that a synthetic approach, opposed to an analytical one, can be an interesting strategy to study this kind of complex system, and computer simulations can have an important role in our attempts to design, model and experiment with selforganizing systems.

Acknowledgments - The authors thank the Brazilian National Research Council (CNPQ), the Graduate Personnel Improvement Coordination (CAPES) and the State of São Paulo Research Foundation (FAPESP).

\section{REFERENCES}

[1] J. Queiroz and C. N. El-Hani, "Towards a multi-level approach to the emergence of semiosis in semiotic systems," Technical Reports DCA-FEEC-UNICAMP, DCA04-07, 2004.

[2] A. Gomes, R. Gudwin and J. Queiroz, 'Towards Meaning Processes in Computers from Peircean Semiotics," S.E.E.D. Journal -- Semiotics, Evolution, Energy, and Development 3 (2), 69-79, 2003.

[3] C. S. Peirce, The Essential Peirce: selected philosophical writings, Vol. 2, Peirce Edition Project (Ed.), Bloomington: Indiana University Press, 1893-1913. [Quoted as EP2, followed by page number]

[4] C. S. Peirce, The Collected Papers of Charles S. Peirce, Vols. I-VI, C. Hartshorne and P. Weiss (Eds.), Cambridge: Harvard University, 1931-1935. [Quoted as CP, followed by volume and paragraph number]

[6] A. Stephan, "Varieties of Emergence in Artificial and Natural Systems," Zeitschrift für Naturforschung 53c, 639656, 1998.

[7] A. Stephan, Emergenz: Von der Unvorhersagbarkeit zur Selbstorganisation, Dresden and München: Dresden University Press, 1999.

[8] J. Fetzer, "Thinking and Computing: Computers as Special Kinds of Signs," Minds and Machines 7, 345-364, 1997.

[9] A. Gomes, C. N. El-Hani, R. Gudwin and J. Queiroz, "Towards the emergence of meaning processes in computers from Peircean semiotics", Proceedings of the European Computing and Philosophy Conference, E-CAP 2004, Pavia, Italy, forthcoming.

[10] S. N. Salthe, Evolving Hierarchical Systems: Their Structure and Representation, New York: Columbia University Press, 1985.

[11] A. Loula, R. Gudwin and J. Queiroz, "Symbolic Communication in Artificial Creatures: an experiment in Artificial Life," Lecture Notes in Artificial Intelligence, 3171, 336-345, 2004. 